\title{
Grandeur et décadence des nouveaux centres urbains
}

\section{Laurent Devisme}

D anslesannées 1960 la conscience d'une crise du référent spatial de la ville se conjugue avec la remobilisation de sa figure centrale. D escolloquesen E urope abordent la question des centres. Le VIII ${ }^{\mathrm{C}}$ CIAM d'H oddesdon (1951) était déjà consacré au thème The core of the city et les groupes d'architectes néerlandais et anglais pointaient l'acuité du problème du centre identifié comme l'âme de la ville en train de se déliter. Ces premierséléments de critique du dogme fonctionnaliste au sein du milieu architectural n'ont trouvé de l'ampleur qu'un peu plustard dansd'autresmilieux. En 1966, au Congrès d'Amsterdam intituléU rban coreand inner city, leschercheurs européensne remettaient pasen cause la primautédu centre comme organe majeur. Ilsinsistaient même en le chargeant du rôle d'accueil desfonctions et deséquipements d'un niveau élevé. En 1969 le colloque de l'association «U rbanisme et commerce» à Stockholm titre sur l'inquiétude fondamentale : «Le centre des villesa-t-il encore un avenir?».

\section{Le problème des centres dans l'humanisme scientifique}

Les années 1960-1970 ont été marquées en France par une importante « réflexion opérationnelle » sur la centralité. La question descentresurbainss'estmanifestée par la montée en puissance d'interrogations travaillantle pol itique au niveau national et dont s'est saisi, logiquement, I'appareil de commande de recherche. L'élément qui peut servir de révélateur tardif du " problème des centres », objectivé dès lesannées 1960, est le premier grand appel d'offres de recherche urbaine lancé par le Comité urbanisation du vi ${ }^{\mathrm{e}} \mathrm{Plan}$ en 1971. Quatre tomesontété publiésà lasuite destravaux préparatoires, destinés à « fournir des éléments de réflexion aux autorités chargées de la politique urbaine. » (Commissariat G énéral du Plan, 1970, préambule du premier tome) ${ }^{1}$. L'ensemble des recherches est essentiellement préoccupé par les dimensions de l'urbanisation et par son rythme. L a ville est vue comme facteur du progrès de la civilisation et en même temps comme défi face àce progrès: leshommesseront-ilscapables de la maîtriser pour y vivre? Alors que les attentions cognitives sont tournées vers lesstatistiques, lesattentions pratiques relèvent de l'aménagement dans son acception classique consistant dans la répartition des fruits de la croissance, dans I'organisation et la rationalisation de l'espace.

Quant à la « question des centres»: « Pour nous, sur la mort des centres anciens, sont en train de naître des centres nouveaux», (C ommissariat Général du Plan, 1970-a, p. 101). Le constat de la morts'établit par la mention des deuxphénomènes que sont l'évol ution des moyens de communication et le déploiement du secteur tertiaire : «En réalité, leproblème des centres n'est pas seulement un problème sentimental (garder nos bonsvieux centres auréolés de gloire), ce qui ne serait pas, àce titre, si méprisable. C'est un problème capital de l'économie moderne : production, consommation, échanges, dépendent pour une large part del'action énergique que nous saurons mener pour réaménager nos centres principaux et créer nos centres secondaires», (Id. ibid.).

Le deuxièmetome, intitulé «La sociétéurbaine » consacre son dernier chapitreà «l'appartenanceà une culture urbaine » définie par la communication etl'échange. Y est reconnueà cet égard la fonction des centres, une fonction ancestral e de point de rencontre et une fonction de « catalyseur » pour des populationsarrivant de toushorizonssansréseaux de relations préexistantes, déracinés pour ainsi dire : « $M$ ais sans doute convient-il de ne pas se référer à une conception rétrospective des relations urbaines, où la ville serait une collection de villages », (C ommissariat général du plan, 1970-b, p. 87). Satisfaction des besoins, animation desvillessont desleitmotive de l'époque. L'optimisme est de règle, ce qu'illustre par exemple l'affirmation que l'on ne peut plus confondre M ourenx avec Bagnols-sur-C èze, M assy avec Sarcelles, au motif que des sociétés local es y ont pris vie : le tempsfait son

1. C hangement d'inflexion pour le Plan qui ne parle plus seulement de planification nationale.

Les Annales de la rechercheurbainen ${ }^{\circ} 98$, 0180-930-IX-05/98/pp.25-33 @ M TE TM 
œuvre. C et effet magique de la sédimentation temporelle est toutefois couplé avec I'apologie d'un interventionnisme permettant d'éviter la suburbanisation àl'américaine.

N ous voyonsàl'œuvre une combinaison de rationalisme et d'humanisme scientifique ${ }^{2}$. D eux ansaprèsla publication du $D$ roit à la ville d'H enri L efebvre, les planificateurs se permettent la mention du livre en post-scriptum, parlant d'une critique impitoyable qui pourrait servir « d'utile décapant aux hommes de recherche et d'action », (C ommissariat G énéral du Plan, 1970-b, p. 151). D 'un côté desutopiesurbainessont appelées, del'autreil convient d'adapter la ville auxnouvelles contrainteséconomiqueset technologiques. Comments'articulent ces deux faces? Comment donner à voir ce que l'on peut appeler l'utopie technocratique du polycentrisme et identifier des lignes de partage au sein de cette utopie? Plusieurs numéros de la revue U rbanisme au cours de ces annéesmontrent, au-delà de la diversité apparente des dossiers traités, un état d'esprittraduit par le partage d'un lexique et de références.

Cetterevue engage àl'époque desproposde professionnels, de «chercheursopérationnels», d'hommes politiques, servant égal ement de chambre d'écho aux discussions dela recherche urbaine, leur donnantun prolongement dansle monde professionnel. La plupart desmembresdu comité de rédaction sont impliqués dansl'urbanisme à titre d'expert ou de responsable de grande opération d'urbanisme. Les dossiers de la revue impliquent ainsi un ensemble de points de vue qui en font un deslieux privilégiés d'inscription del'esprit del'urbanisme. Autrement dit, elle exprime la normativité en actes de l'urbanisme avec l'exposé de cas édifiants au sein d'un dossier exprimant l'état des choses. Q ue donne-t-elle à lire ? ${ }^{3}$.

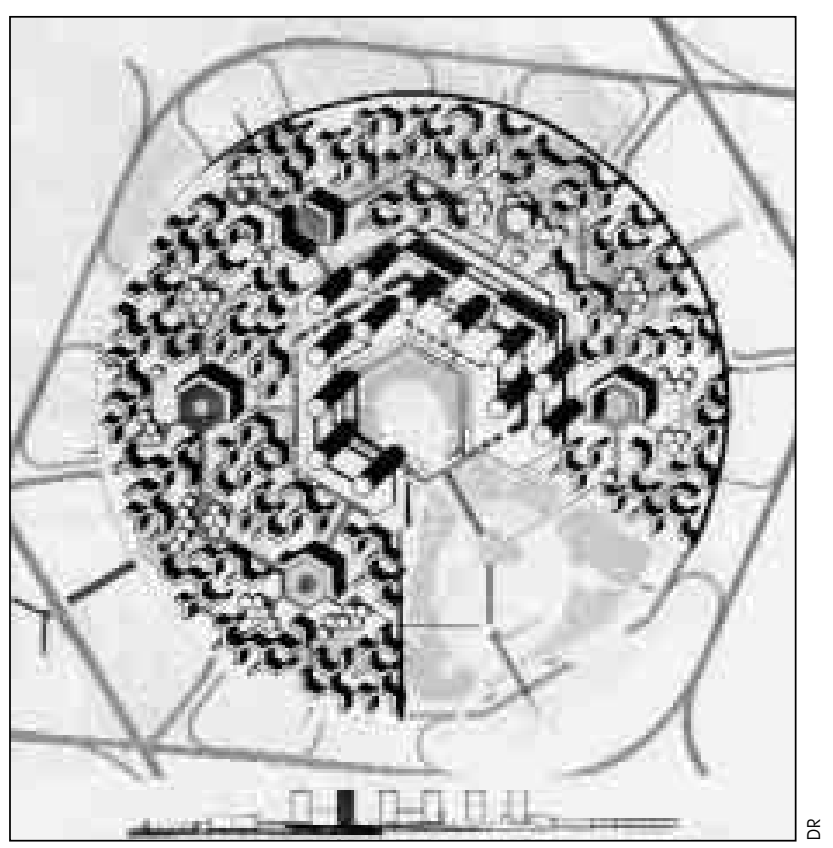

L’urbanisme des nucléoles, in Hervé Mathieu, René Magnan, Orthopôles, villes en îles, Paris, CRU, p. 169. Où l'on voit une parfaite superstructure

\section{L'utopie technocratique du polycentrisme et ses variations}

Le dossier du n 99 (1967) est précisément consacré aux « centres urbains ». Sa tonalité est donnée par un éditorial collectif évoquant le dénuement desfils de la vie urbaine. Le «C entre Foyer » est en crise : «L e fait que le déplacement de certaines fonctions semble obéir aux lois de l'économie qui conduisent l'explosion urbaine vers un étalement et un éclatement, n'apporte cependant pas la preuve que la notion de Centre-Ville ait perdu son caractère sacré et que ce ne soit plus qu'une machine dont l'existence montre l'utilité sans apporter la démonstration de sa nécessité », (éditorial, p. 3).

Une photographie de la maquetted'Auroville, ville expérimentale devant se construire « auxIndes», destinée à recevoir 50000 habitants, vient en appui avec une légende érigeant sa mission dansla preuve que «la spiritualité etlestechniques lesplusmodernesne sont pas incompatibles» ${ }^{4}$. Un « état des connaissancesen F rance et à l'étranger » est d'abord proposé par C harles D elfante, qui fait référence à l'école américaine de géographie urbaine parlant de «l'agonie des centres »: «Aussi, cette dégénérescence descentresurbains, processus naturel obéissantà la loi du moindre effort, appelle-t-elle des actions radicales et urgentes, fort éloignées de tout protectionnisme ou mesuresd'interdiction. Aussi, capitales et grandes villes abordent-ellesleur modernisation en faisant porter leurs efforts sur leur centre, afin d'exprimer leur vocation de métropole nationale ou régionale », (p. 5). Le paragraphe consacré à la circulation, en écho au rapport Buchanan ${ }^{5}$, est celui le pluscatégorique à propos de l'accessibilité : la villene peutêtre un centreattractif qu'àla condition de pouvoir absorber et ordonner lesvolumes croissants de circulation, cequi suppose hiérarchisation et ségrégation des flux : stationnement en souterrain et piétons seuls dans le centre. La conclusion de I'article ouvre aux innovations formelles sans lesquelles « la vieen ville risquerait dene plusavoir de raison d'être» : «ville spatiale», «cité al pha », « ville entonnoir »...

Le centre comme lieu de rencontre vivante des âges successifs de la cité doit donc s'adapter à la ville à venir dont les appellations sont clairementfuturistes. D ansun textedont

\section{Cf. D evisme, 2005, chapitre I pour approfondir.}

3. Six numéros ont été analysés en détail, relevant d'une même série : à propos du Plan d'Aménagement et d'O rganisation Générale (PAD O G) de la région parisienne $\left(n^{\circ} 68,1960\right)$, des métropoles d'équilibre $\left(n^{\circ} 89,1965\right)$, desutopies urbaines et visions futuristes ( $\left.n^{\circ} 92,1966\right)$, des centres urbains ( $\left.{ }^{\circ} 99,1967\right)$, des créations urbaines $\left(n^{\circ} 102-103,1967\right)$ et enfin des villes nouvelles françaises $\left(n^{\circ} 114\right.$, 1969). À la même époque, on peut aussi se référer à la revue $L$ a vie urbaine qui est al ors plutôt une revue professorale, « organe de I'institut d'urbanisme de l'U niversité de Paris » dirigé par Pierre Lavedan.

4. Auroville, à $10 \mathrm{~km}$ de Pondichéry, fut inaugurée en 1968. Elle était censée devenir ville de la fraternité humaine à l'échelle mondiale.

5. Traduit en France en 1965 et publié à l'imprimerie nationale sousle titre L'automobile et la ville. 


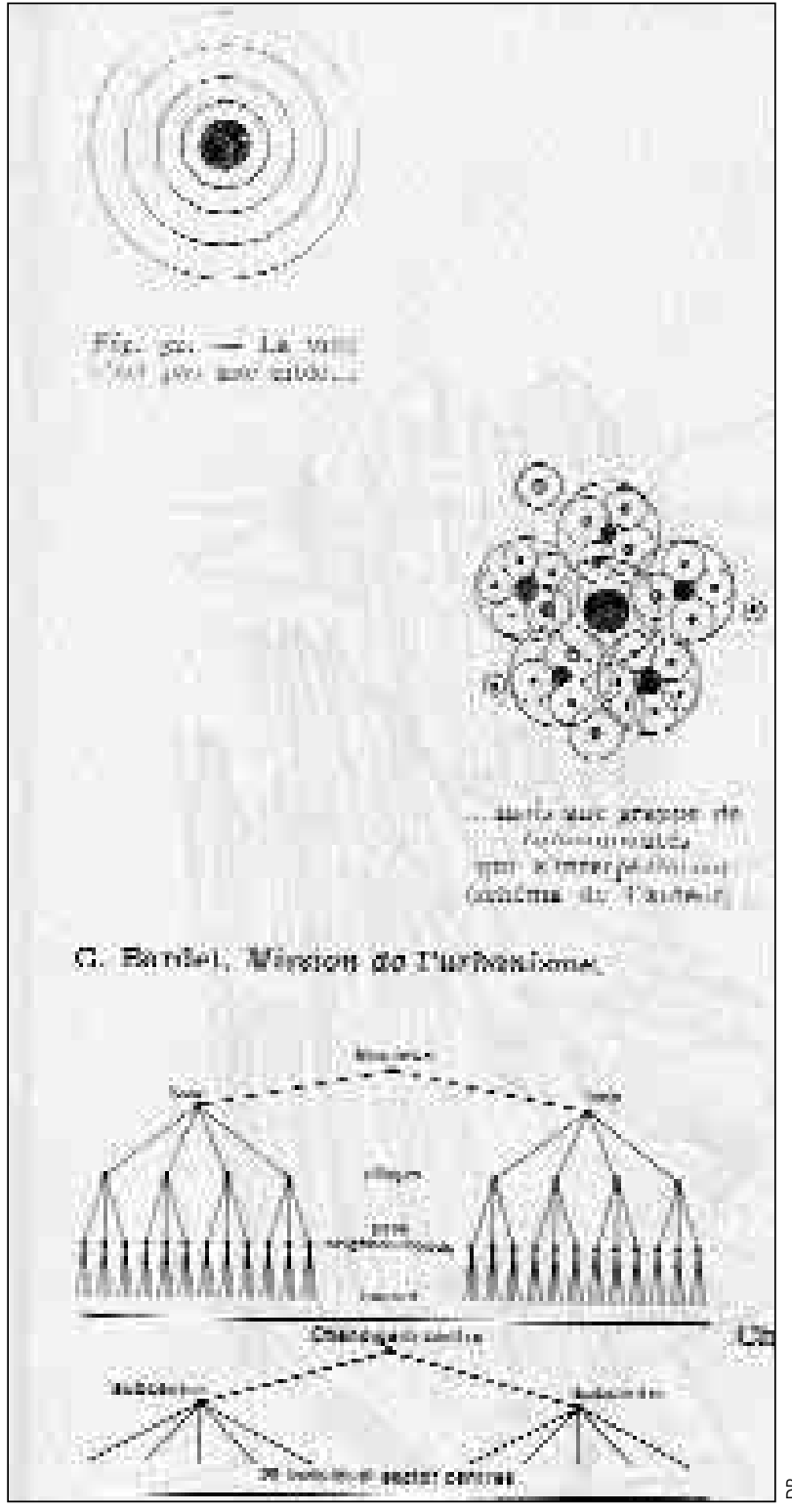

«Villes de papier » de Jean-Paul Jungmann, Utopie, n 1, 1967. L'auteur juxtapose les schémas alors en vigueur de C. Alexander et de G. Bardet

l'esprit est proche de celui écrit par Olivier G uichard pour introduire lesmétropoles d'équilibre (et pour cause, son auteur est délégué adjoint à la DATAR), Jérôme $M$ onod ${ }^{6}$ traite de I'«Influence régionale descentres urbains» dansle plus pur style planificateur : «Au moment où vouscherchez la réponse au 1/10 000 ou même au 1/50 000 dansle centre des villes, la force descentresurbainsse manifeste au 1/1 000000 àl'échelle de la F rance. [... ] Faites nous de bonnes villes, nous vous ferons de bonnes régions », (p. 18). Sur fond des plans de Chandigarh et de Brasilia, I'auteur justifie le rôle du groupe central de planification urbaine, évoquela « décentral isation tertiaire » et l'animation régionale comme conséquences directes d'une réflexion sur les centres urbains. La mise en avant des aires métropolitaines est en même temps celle de leurs promoteursqui ont une mission forted'institution territoriale. M onod fait référence aux travaux du $V^{e}$ Plan montrant que la politique met trop l'accent sur l'urbanisation périphérique : il convient de réinvestir politiquement les centres urbains.

Ainsi se justifie l'article d'un chargé de mission au Commissariat général du Plan pour évoquer «Les centres urbainset les programmes d'équipement». Pierre M azzolini écrit, par rapportau ve Plan en cours : «Partant d'un schéma radioconcentrique, sans doute adapté aux premières étapes de la croissance, il faut maintenant répartir l'initiative de la croissance, la déléguer à de nouveaux pôles, tout en donnant aux centres traditionnels des atouts pour faire face à une concurrence plusactive entre centres de décisions», (p. 22).

La délocalisation-rel ocal isation des entreprises de service (nommément les supermarchés), suivant le mouvement d'une clientèle spatialement centrifuge, produitun désordre spatial auquel il faut remédier. L'Amérique est sur le devant de la scène avec un long article d'un planificateur mythique pour les promoteurs de centres commerciaux, en l'occurrence Victor G ruen 7. Le planificateur M aurice Rotival parle du centre comme lieu de rencontres maisaussi comme lieu du commandement de la ville par le contrôle électronique de ses activités et mouvements ${ }^{8}$.

Les hommes politiques sont fortement présents dans ce numéro avec notamment Edgar Pisani, ancien ministre de I'É quipement, mais aussi Jean Royer (le maire de Tours) qui épouse le modèle du centre directionnel. Le long dossier débouchesur uneréhabilitation de la C ité, du C entreU rbain (les majuscules sont utilisées par l'architecte L ouis $\mathrm{G}$ eorges N oviant) et sur « la logiquedu non quantifiable», titre du texte de $M$ aurice-F rançois Rouge, qui propose une synthèse $d u$ Congrèssur lecentre desvilles d'Amsterdam et du $17^{\circ}$ Jour de I'urbanisme : c'est singulièrement en ces lieux (centraux) apprend-on, que la vie et l'animation doivent émaner de la matière. La central ité est liée aux notions d'intensité et de concentration.

Ce dossier fait part d'inquiétudes, de conceptions et de débats communs : le centre que l'on connaît (ou croit connaître) est en péril ; il faut l'adapter (en le modernisant

6. Jérôme M onod connaît ensuite un parcours de compagnon de route de Jacques C hirac, à son cabinet de premier M inistre en 1975, au secrétariat général du RPR et comme conseiller du Président depuis 2000. II fut entre tempsPD G de la L yonnaise desE aux.

7. Véritable gourou, il est ainsi évoqué par Jean-L ouis Solal, promoteur des centres commerciaux régionaux en France - dont celui d'É vry : "L'urbanisme avait à ses yeux pour fonction de faire vivre les gens ensemble et que toute forme de ségrégation des activités ne pouvait que les séparer. II nous a convaincus avec les urbanistes de la ville nouvelle de construire É vry en favorisant la mixité d'usages, le brassage des populations et le chevauchement d'activités», (Solal, entretien avec F. M ichel, Archi C rée ${ }^{\circ} 275,1998$ ).

8. Comment ne pas penser à «Alpha 60 », cette ville futuriste sujet du film de Jean-Luc G odard, Alphaville - une étrange aventure de L emmy C aution (1965) régie par un tel contrôle? 
radicalement ou en le protégeanten proposant d'autrescentralités). II représente un prisme d'analyse de la société urbaine.

$M$ ax Stern, conseiller technique au ministère de l'équipement et du logement présente dans le $n^{\circ} 114$ (1969) les villesnouvelles comme une réponse vigoureuse à une poussée d'urbanisation tard venue. C ette réaction est inédite, les vieux centres ayant « toujours su s'adapter »; on pourrait certes ne rien prévoir d'autre qu'organiser au mieux la rénovation urbaine et jalonner les banlieues de quelques grands ensembles; " la ville nouvelle au contraire, conséquence et clé de voûte du schéma directeur assume, en des sites convenablement choisis, un ensemble de fonctions. Elle fixe une part desfoyers qui se di sperserai ent au hasard des possi bilités dans l'agglomération, elle soulage sans les concurrencer lescentres desvilles, elleébauche un nouveau genre de vie où le déploiement dans l'espace des grandes fonctions urbaines tient compte des nouveaux moyens de déplacement individuelset collectifs», (p. 4). D e vraiesvilles doivent voir le jour, avec un centre qui soit administratif, culturel et commercial. Accompagnant la liste des villes nouvelles officielles, deux cas sont présentés dansla revue : Toulouse-Le M irail et le centre commercial régional de Parly 2. Le dispositif de Parly est misen avant avec la théorie américaine des magnets : deux grandsmagasins aux extrémités du mall organisent les va-et-vient des clients.

L'évocation de ces deux dossiers permet de se faire une idée des convergences lexicales et de la circulation des catégories sémantiques en vigueur mais montre aussi des divergences, dansl'ensemble peu explicitées.

\section{Forme polycentrique et questions d'échelle}

U ne vision à la fois polycentrique ethiérarchique, hantée par l'image du centre directionnel, domine dansla production desvillesnouvelles. Lorsquele C entre de Recherche d'U rbanisme publie en 19750 rthopôles. Villes en îles, il valorise un modèle polycentrique avec un principe centre/périphérie très prononcé. Si le paradigme dominant de la planification dans ces années est celui de l'équilibre et du rééquilibrage, il passe par des créations de centres tertiaires avec des effets d'entraînement que cherchent à théoriser les travaux dominants de l'économie urbaine. La conception de la central ité dansun contexte de fantasmessur la sociététertiaire peutêtre aujourd'hui repérée en plusieursoccurrencesgéographiques: à Lyon, où l'opération Part-D ieu n'estni une création extra-urbaine ni une mutation sur place par extension du centre ; à Bordeaux où se créé le quartier d'affaires dit de $M$ ériadeck, à $M$ arseille avec l'opération de la Bourse-Sainte Barbe ; au sud d'O rléans avec l'opération de la Source, à Rouen avec Saint-Sever... D esvilles de moindre importance dansl'armature urbaine sont également concernées.

Si l'accord est assez général sur le polycentrisme, certains voient cette solution comme général isable à l'ensemble des aggl omérations (il faudrait passer du paradigme du plan radioconcentrique à celui du polycentrisme) et d'autres indiquent desseuilsvoire des réservessur le volontarismenécessai re pour créer cesnouveauxnoyaux. Surtout, cette question pose celle desmargesdemanœuvre, desdisponibilitésfoncièreset renvoie demanière plusthéoriqueàla discussion surle «continuisme» ou la discontinuitéspatiale. L a plupart desurbanistess'expriment sur cette question. D ansdes approchescomparatives, avec les new towns anglaises notamment, certains pointent la trop grande proximité desvillesnouvellesà Pariset suggèrent qu'un éloignement plusgrand leur aurait assuré davantage de vitalité ou d'animation. D 'autresen revanche voient déjà trop d'écartementen France et auraient plutôt penché pour desaccroches spatiales plus immédiates, en continuité. M ais encore fallaitil contourner l'opposition politique de la ceinture rouge et trouver desespacesmoinsurbanisésdonc moins chargés pour déployer desformulestrès volontaristes.

Si la question des nouveaux centres revient à toutes les échelles d'aménagement, certains maintiennent l'idée d'une hiérarchieurbaine (pour structurer lesrégionsnotamment) et d'autres critiquent plus ouvertement le système pyramidal en espérant voir se concrétiser un espace polycentrique non hiérarchique. La première optique cadre avec la philosophie des métropoles d'équilibre et le credo d'innerver la région urbaine voirel'arrière paysà l'aide de centres directionnelset « centres directionnels bis » ou « centres relais ». La seconde, plus ou moinsinspirée par desthéories critiques de la colonisation du monde vécu par la bureaucratie, imagine de l'urbain partout, l'abolition du schéma centre/périphérie ou bien, plus prosailquement, commence à douter de la possibilité de polariser volontairement les espaces périphériques, entérinant, sans I'objectiver de cettemanière, la villesectorisée décrite récemment par D avid M angin (2004). Q uelques « optimistes » (et les numéros92 et 102-103 d'U rbanismetémoignent deleur nombre) envisagent dansla perspective d'un urbanisme spatial, futuriste, des « noyaux urbains» sansrapport avec lescontenusreconnus auxcentresexistants. C ritiquantl'idée du continuisme, ilsoptent pour la discontinuité et incarnent souvent le progrès par des incarnations de centres d'activités intellectuelles.

Plusieurs échelles d'intervention sont concernées dans les années 1960 : I'espace urbain hérité à protéger, l'espace régional à organiser, le territoire métropolitain à équilibrer. C ette mobilisation multiscalaire vise à la foisle maintien des centralités existanteset la création de nouveaux lieux centraux. La croyance dans la possi bilité de dupliquer la centralité est forte. Lestroiséchellesmobilisées en même tempsméritent que l'on s'y attarde car ces niveaux d'intervention sont loin d'être convergents mêmesi, dansles discourstechnocratiques de l'époque, ils sont parfai tement complémentaires.

Que ce soit dans le SD AU de la Région Parisienne ou bien danslesschémas directeurs desmétropoles d'équilibre, la planification urbaine est taraudée par la question d'un changement d'échelle, motivant souvent l'idée de centre directionnel à créer de toutes pièces. $C$ ette figure, le centre directionnel, est : au service de la M étropole d'équilibre, elle- 
même au service de la régionalisation, elle-mêmeau service de la modernisation du pays.

Le credo moderniste, grand projet unificateur, est téléologique. À partir desfocal isationsspatiales, un processusd'irrigation est imaginé, sel on un schéma radioconcentrique de diffusion de l'innovation. M ais, selon les échelles impliquées, ce que recouvre la central ité n'est pas du tout du même ordre. Et des emboîtements vertueux ici rappelés, on passe à des contenus pouvant être fort différents voire antagonistes. S'agit-il en effet de rassembler desélémentsdispersésafin de créer del'urbanité - appel qui se fait de plus en plus insistant dans les années 1970 sousla bannière de l'animation urbaine ? S'agit-il plutôt de desservir des populations ayant tendance à se disperser, d'accompagner de nouvelles polarités marchandes dont on perçoit qu'elles ne seront pas résiduelles? La même forme appellealorsdescontenusbien différents. Commel'écritDaniel Béhar, «on prononce "villesnouvelles" maisça s'écrit "centres urbainsnouveaux"». Autrement dit, le terme de "ville" entretient la confusion volontaire entre deux conceptions del'objet. Une conception volontariste, consistantà créer, àl'anglaise, des villesautonomes, dotéesd'un ensemble complet de fonctions, susceptibles d'accueillir une population importante; une conception "del ouvrieriste" consistantà concevoir descentres urbains destinés, pour partie, à accueillir de la population nouvellemaissurtout à desservir par lesemploiset lesservices, une population croissante », (Béhar D ., 2004).

\section{La part du symbolique}

Q uelquesauteursne sont pasloin d'exprimer ce qu'écrit Debord, sansappel, en 1967, lorsqu'il résume ainsi la devise desvillesnouvelles: «Ici mêmeil n'arrivera jamaisrien et rien n'y estjamaisarrivé » (D ebord G ., 1967, § 177) au motif que les processus d'aménagement du territoi re se résument à la dislocation descentresanciens par lesautoroutes età l'accompagnement de nouvelles polarisations autour des usines de distribution ${ }^{9}$. C hez plusieurs urbanistes et chercheurs, le rappel de la dimension politique induite par la centralité charge lourdement sa val eur spatiale.

Au demeurant, quel quesannéesplustard, lorsque Samuel Bordreuil etSylvia O strowetsky conceptualisentle centre dans la ville contemporaine, ils le considèrent au départ, dansune théoriegénérale de la communication, comme infra-expressif, l'espace n'étant plus chargé de symboliser ce qu'il contient. La crise de l'espace estal orscelle dela modernitéet la réponse postmoderne ne convainc pas dans la mesure où elle relève largementd'un projet nostal gique : retour au moment précédant la séparation de l'homme et de son œuvre. Si l'on accepte ce postulat selon lequel le centre est un « dispositif de synthèse représentative », alors l'anthropologie des mondes contemporainsneferait que confirmer sa disparition. Sansactiver cette hypothèse, signal onsque c'est la multiplication ou la fragmentation du centre qui est le plus souvent ignorée. L orsqu'on

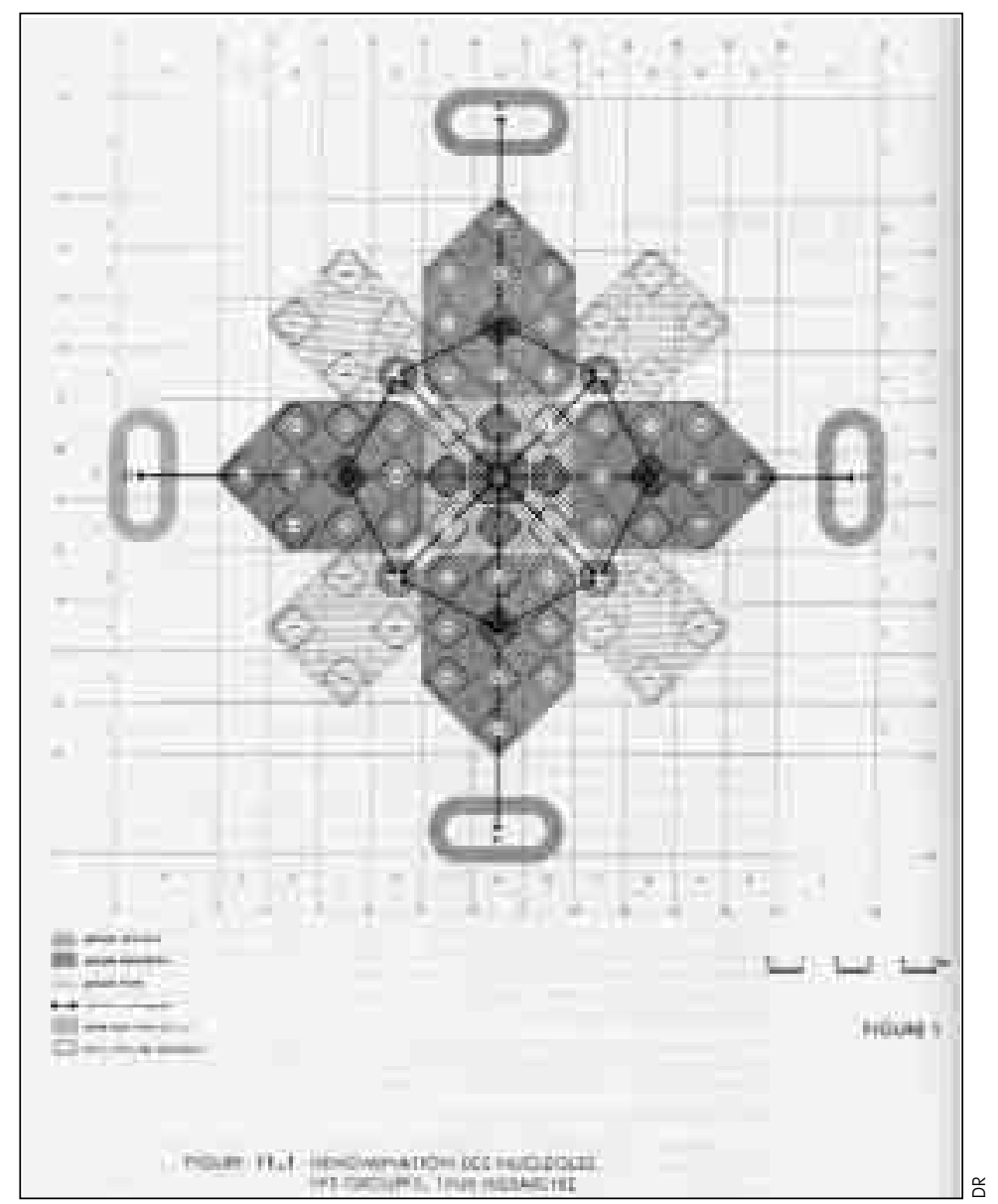

Orthopoles, une vue générale, op. cit., p. 118. Grille et répartition des nucléoles. Apologie du quadrillage à l'heure d'une reconsidération du métabolisme urbain. On lit dans l’avertissement que le mouvement de contestation de Mai 68 s'est naturellement attaqué, entre autres objectifs «à ce produit (en même temps que condition) de l'évolution de la civilisation que sont les villes », (p. 5).

parle d'autres centres que le « premier», ilssont toujours vus en creux, comme des pôlescertesmaisnon descentres.

L'analyse thématique et géographique de la « crise des centres» permet de poursuivrel'étude dela construction sociale du phénomène danslesannées 1960, 1970. Cetteconstitution du problème est inséparable d'une conscience réformatrice des espaces habités. D 'où la récurrence d'une présence du volontarisme politique danslesdescriptions elles-mêmes. Les formes de ce vol ontarisme peu vent différer, entre héritagesdu social isme municipal et É tat aménageur ; elles peuvent aussi coexister. Si l'É tat a général ement une action prépondérante sur les territoires dans les années 1960-1970, les deux cas de Grenoble et de Toulouse montrent toutefoisla part du local dans I'orientation de certaines décisions : les maires D ubedout (G renoble) etBazerque (Toulouse) ont su capter l'opportunité d'une production urbaine sans précédent en termes quanti-

9. C hez les situationnistes, la centralité a généralement une double connotation : ludique et nostal gique. 


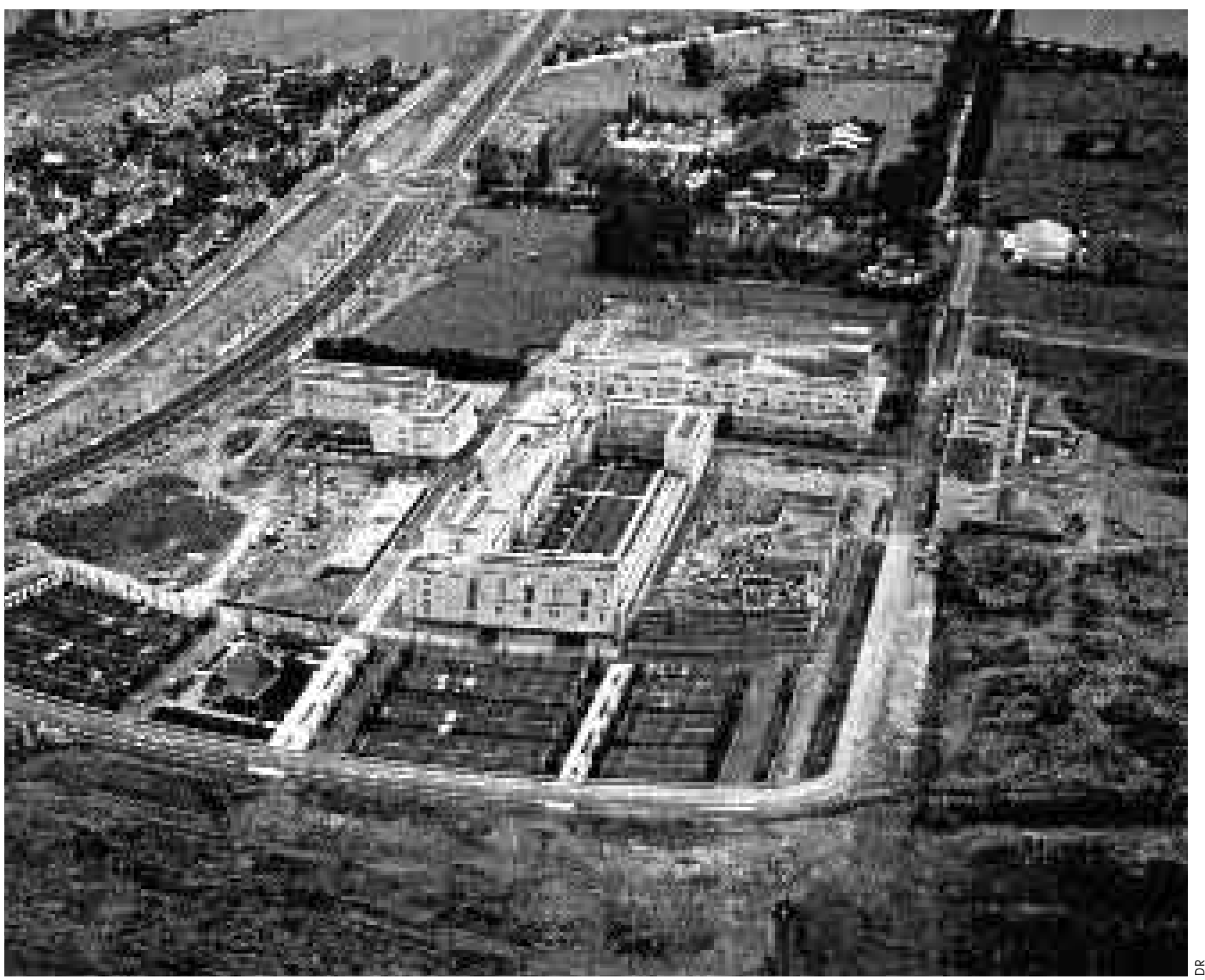

Construction d’un centre urbain dans les années 1990 : Saint-Jacques-de-la-Lande centre, vu du ciel. Source AMC, Le Moniteur Architecture, avril 2000

tatifs; ilsont ensuite cherché une certaine maîtrise desformes et des processus engagés. D e surcroît, certaines communes périphériques de ces agglomérations ont pu constituer rapidementun champ d'action urbaine (Échirolles, Colomiers).

\section{Le devenir politique de la forme centralité}

Les équipes d'urbanisme qui ont contribué à l'advenue de nouveaux centres urbains ont la plupart du temps tiré les leçons à la fois des méfaits de l'urbanisme colonial et des défauts de la programmation des grands ensembles. C es équipesont souvent cherchéla mise en place d'un « urbanisme anthropologique» (Brossaud C ., 2003) qui lespousse parfois à se situer en opposition relative à l'É tat jacobin ${ }^{10}$.

Certains territoires communaux relevant des premières couronnes d'aggl omération ont connu des projets de constitution de nouveaux centres, nouvelles villes voulues par des équipesmunicipales. L'évolution de cesprojetstémoignedans certainscasd'unetension territorial e opposée à celle des Villes nouvelles. Alors que I'histoire de ces dernières peut être lue comme une territorial isation progressive jusqu'àl'entrée dans ledroitcommun, lesprojetsque nousévoqueronsici montrent la conscience croissante d'un fonctionnement urbain tout compte fait peu lié au périmètre du territoire communal, multicentrique à souhait dans une urbanisation dispersée. C ertes les nouvel les central ités en territoire périphérique suiventun modèle d'organisation del'espace proche de celui desvillesnouvellesétatiques. M aiscesprojetssont d'autant plus intéressants qu'ils ne mobilisent pasl'appareil d'É tat comme les villes nouvelles. Par exemple à Toulouse, l'É tat s'investit prioritairement dansla ZUP du M irail qui s'érige à moinsde cinq kilomètres de «Colomiers Ville N euve » : les deux

10. On retrouve cette idée ayant inspiré le titre de l'ouvrage de M . Amiot, L es sociologues contrel'É tat : à la fois en opposition et « tout contre ».. 
démarches se mènent en parallèle, C olomiers à l'ombre des projets médiatisés de $\mathrm{C}$ andilis, Josic et Woods. H érouville-Saint-C lair relève certes d'une ZUP au départ, mais, en dehors de la ville-centre et dansune commune dont les élus vont vite revendiquer une certaine autonomie (via le mouvement des $G$ roupes d'Action M unicipale).

D eux enjeux se sont présentés si multanément dans les années 1960, 1970 pour ces communes périphériques: l'affirmation d'un territoire politique et la construction d'une vie urbaine à part entière, dense et diverse, c'est-à-dire centrale (ce qui est davantage que le si mple «équipement » de la banlieue). L a co-occurrence de ces enjeux amène à la volonté de traiter l'un par l'autre et à vouloir que la forme urbaine tienne lieu d'identité, pour tous ces nouveaux habitants, ex-ruraux, immigrés de l'intérieur ou de l'extérieur, « horsains » comme on dit en Basse-N ormandie... À la Villeneuve de G renoble-É chirolles, à H érouville-Saint-C lair, à Colomiers et à Saint-Jean-de-Braye dans une moindre mesure, il est bien question de cela, d'une socialisation spécifique passant par l'expérimentation formelleà l'échelle urbaine. L'urbanisme à pensée forte (C hal asY., 2000) revient à un ensemble de convictions efficaces lorsqu'il est lié au social isme municipal ${ }^{11}$. D ans cette orientation certaines formes peuvent produire d'autres modes de sociabilité : les thèmes de la rencontre, de la promenade, de la flânerie sont alors déclinés par le biais des réflexions sur la central ité urbaine. Elles se situent la plupart du temps dansle sillage d'une pensée urbaine comme celle d'H enri Lefebvre, la centralité étant une forme pouvant appeler d'autres contenus.

C es projections en partie fantasmatiques ont décliné. D e surcroît, l'idée de pouvoir créer d'autres types de villes, issue d'une pensée utopienne, a laissé la place à d'autres préoccupations ou à un retour nostalgique à des formes urbaines éprouvées - ce que le vaste mouvement des parcs résidentiels illustre souvent. Le déplacement des centres d'attraction (vs centres d'organisation) a posé de nouvelles questions.

Cependant, le déclin de cette posture ne s'est pas accompagné d'un abandon de la référence à la centralité. La version opérationnelle de la quête contemporaine de centralité passe par la réalisation de ZAC de centre urbain dans certai nescommunes. Les principaux objectifs sont souvent de favoriser l'accessibilité au «Q uartier C entre », compléter et reval oriser les lieux de centralité du quartier, renforcer les fonctions urbaines complémentaires et ainsi permettre I'accueil de nouveaux commerces et services, logementset équipements... Lorsque l'idéal de la mixité est érigé en objectif, c'est souvent pour rechercher la venue de classes moyennes, les opérations d'urbanisme des années 19601970 ayant été largement supportées par des programmes de logements sociaux dans des visées d'accessibilité sociale élargie. À Colomiers comme à Saint-Jean-de-Braye, on peut lire la dernière étape de projection pour le centre comme la recherche de nouveaux investissements résidentiels pour des catégories sociales plus privilégiées.

\section{La centralité projetée dans la ville multipolaire}

Lestermes de « centre secondaire», de « ville satellite », ont laissé la place à celui de centralité - à créer (rarement), à compléter et achever (plus fréquemment). O utre la corrélation persistante avec le souci identitaire ${ }^{12}$, la central ité est associée à l'esprit de la ville durable, contribuant à une économie d'espace. II ne s'agit plus de limiter l'engorgement des centres existants en créant de nouvellescentral ités, mais bien de différer la dispersion, suite au développement de l'urbanisation en extension où les forces centrifuges sont dominantes. $\mathrm{M}$ ais les communes de première couronne d'aggl omération engagées aujourd'hui dans des projets de structuration de centralité ne sont pas à l'unisson, derrière l'apparat d'un vocabulaire partagé relevant de «la ville sur ellemême ». Ellesont surtout à transiger avec de réellesrelations de contrariété dont les troissuivantes:

- Si la centralité repose sur une maximisation du couplage densité/diversité, cette orientation entre souvent en conflit avec un véritable modèle territorial qui valorise l'idée de campagne et toutcequi lui est associé dansune carte mythique constituant autant de ressources pour lespromoteurs/constructeurs. Leschoix urbanistiques de certaines communesjouent sur les deux tableaux avec un brouillage certain quant à la clarté des orientations.

- Si l'intensité urbaine dépend d'une forte accessibilité, des débats se poursuivent sur sa nature, elle-même dépendante des fonctions accueillies par l'espace central. La centralité commerciale implique tout le temps majoritairementl'unité véhiculaire qu'estl'automobile. Commentse concilie-t-elle avec les autres métriques?

- Une fois le statut de centralité de proximité voire de central ité communale atteint, bien des urbanistes reconnaissent l'impossibilité d'entrer en relation de concurrence avec la ville-centre. Le glissement vers la ville multipolaire signifie al ors une mise en réseaux de centralités de différents ordres. Si tous les profits symboliques restent captés par la ville-centre, lesmotivationsurbanistiques se cantonnent àla

\footnotetext{
11. M entionnons à cet égard et pour marquer l'esprit d'une époque, les pratiques expérimentales de la SO DED AT en Seine-Saint-D enis: construisant principal ement dans les communes communistes, cette société cherchait à « construire du beau pour le peuple ». La revue Archivari tenait le rôle de dynamiseur, de lieu d'échanges, de débats, avec la figure de proue de Jean Renaudie, architecte du centre d'I vry. Apologie était al ors faite de la ville de la complexité, de la mixité, de l'imbrication desfonctions expérimentant de nouvelles formes urbaines, de nouveaux types d'espaces intermédiaires censés appeler la rencontre et l'appropriation.
}
12. M ême à Sénart, dansun projet trèséloigné des conceptions centrales des autres villes nouvelles, la question d'un nouveau centre est associée à un souci de toucher des populations de l'agglomération sénartaise, via des références à l'histoire longue, des actions éphémères et un accent paysager prononcé. C ela dit, la réalité du projet « $C$ arré Sénart » est emportée par un centre commercial de « dernière génération ».


gestion d'incohérences d'infrastructures ou se rabattent sur la qualité architecturale à l'échelle des bâtiments.

- C es difficultés rencontrent des options spécifiques. Et leslignesdivergentes précédemment évoquées se retrouvent danslescomparaisons de projets menéset dansl'organisation de chacun d'entre eux. Cela dit, du côté des ressemblances, dans les témoignages oraux de jeunes retraités, on trouve l'enthousiasme d'urbanistes ayant travaillé en des « laboratoires ${ }^{13}$ qui ont pris la forme d'atel iers publics d'architecture et d'urbanisme, tous sur le déclin dans les années 1990.

C ette caractérisation ne doit toutefois pas éclipser quelques cas sortant du tableau d'ensemble, comme cette commune dela première couronne de Rennes, Saint-jacquesde-la-Lande, engagée depuis 1990 dansun projet de création ex nihilo d'un centre. Surtout l'enjeu est revenu dans les années 1990 de central ités d'aggl omération ou de métropole: ainsi de «L yon C onfluence», «île de N antes», «Bordeaux les deux rives»... La métropolisation polycentrique, effective mais différente de celle clamée dans les années 1960, est rapidement venue dérouter les projets de nouvellecentral ité communale. L'échelle d'agglomération voire de métropole est incarnée selon les cas par des espaces réactivant l'idée du quartier d'affaires, la recyclant dansun cadre paysager, en rapport avec des fleuves contournés pendant l'âge d'or de l'aménagement Le niveau d'organisation de cetteéchelle est désormais bien établi.

\section{En finir avec la centralité ?}

L'intention de créer de nouveaux centres urbains dans les territoires périphériquessignalait, il y a quarante ans, des velléités de dépassement d'un état spatial désorganisé et quelque peu atone : les urbanistesn'avaient plus à faire avec une banlieue vue comme espace d'encanaillement (celui, mythifié, de la fin du XIX $x^{e}$ siècle), mais avec un espace de la banalisation, monotone, lieu privilégié d'observation du quotidien dansson aspect répétitif sousl'effet d'une modernité clignotante (L efebvre H., 1960). Le cinéma a du reste bien contribué à cette documentation du passage d'une ville à I'autre: de la banlieue sauvage et fantasmatiqueà un espace périphérique trop net pour être raconté. Le centre urbain, plutôtfilmésur le modenarratif s'oppose al orsà des périphéries qui seraient le terrain du mode documentaire ${ }^{14}$.

Considérer la projection de centralité dans I'urbanisme récent, c'est en faire un analyseur aussi bien cognitif (ce que porteunetellenotion) que géographique (cequelastructuration volontariste de nouveaux centresa changédansl'organisation urbaine) qui permet notamment de poser les conclusions suivantes

- L'importance de la figure du centre peut souvent se résumer à une entente dans le malentendu. Si le centre est largement investi dans les registres d'action politique (à la suite, probablement, du registre religieux), il a égal ementété fortement instrumenté, outillé pour devenir dansles années 1960 un opérateur de changement d'échelle géographique. D ans cette optique, l'impulsion plus ou moins objectivée revientau commandement desactivitésvia lesecteur «tertiaire supérieur » aux qualités assez floues.

- La récurrence du mot «centre» ne saurait faire oublier d'importants changementsquant à ses connotations. Dansle passage descentresurbainsà la centralité, on glisse ainsi d'un dispositif à une qual ité, d'un urbanisme structurant à des projets de révélation. L orsqu'on recherche, dans les centralités d'agglomération actuellement composées, de nouveaux contenus, de nouveaux programmes, le vocable de centre, du statut quasi magique d'embrayeur d'actionsurbanistiques devient de plusen plussynonyme de central ité commerciale avec ses spécialistes appointés.

- L'un desenjeux pointés dans lesannées 1960-1970 dans les villes nouvelles consistait à développer une animation urbaine spécifique et à permettre le déploiement de sociabilités inédites (la thématique de l'appropriation est alors constante). Réactualiser cet enjeu reviendrait à le formuler comme création (ou re-création) de centralité civique dans la ville dispersée. M aisal ors, dansle couple centralité/mobilité, c'est plutôt le second terme qui est à penser d'abord, comme I'a fort bien montré I saac J oseph à propos de M étéor. La centralité devient al ors une qualité migrante, ce qui suppose un fort aggiornamento.

La central ité n'est plus tant à exprimer, à surligner maisà construire pratiquement. Si la central ité d'organisation du niveau métropolitain est encore peu visible, c'est que sa perception manque de prises. À la visibilité symbolique recherchée par le niveau politique, il manque peut-être une visibilité perceptive, ce qui pourrait être un programme renouvelé pour la centralité, à condition d'envisager cette perception en mouvement, dansdesflux toujourscroissantset non en un seul lieu quelle que soitl'économie de sagrandeur...

\section{Laurent Devisme}

13. Le colloque du programme interministériel d'histoire et d'évaluation des villes nouvelles françaises, en avril 2005 était intitulé : Villes nouvelles, laboratoi res d'architecture. 1965-2005. N otons la sortie concomitante d'un numéro d'E spaces et Sociétés à proposdes « Villesnouvelles, trente ans après ».

14. Citons quelques exemples de cette partition cinématographique entre banlieue et périphérie : la banlieue est présente dansun grand nombre de films de Robert G uédiguian avec la (désormais) figure de l'E staque, quartier ouvrier du nord de $M$ arseille (L'argent fait le bonheur, 1993, À la vie, à la mort, 1995); c'est une autre banlieue marseillaise qui est présente dans $U$ n, deux, trois sol eil un film de Bertrand Blier (1993), également réalisateur de Buffet froid (1979), film dont les premières minutes mettent en scène une périphérie plus qu'une banlieue à l'atmosphère pesante (la station de RER, la mort dans un couloir d'accès et les premiers habitants d'une tour de la D éfense, tousinquiétants). É ric Rohmer a quant à lui fait un documentaire sur $C$ ergy-Pontoise et plusieurs de ses films mettent en scène des ménages des villes nouvelles. La périphérie est davantage traquée par un cinéaste comme W im Wenders (Alice dans les villes). 


\section{Références bibliographiques}

Béhar D., (2004), " La ville nouvelle en Île-de-France ou la fortune d'un malentendu ", Pouvoirs locaux, $n^{\circ} 60$.

Boltanski L., Chiapello E., (1999), Le nouvel esprit du capitalisme, Paris, Gallimard.

Bordreuil J.-S., (1987), La production de la centralité urbaine, thèse d’État, Université Toulouse-Le-Mirail.

Brossaud C., (2003), Le Vaudreuil Ville Nouvelle (Val-de-Reuil) et son imaginaire bâtisseur. Identification d'un champ autour d'une ville, Paris, L'Harmattan.

Chalas Y., (2000), L'invention de la ville, Paris, Economica.

Commissariat général du plan, (1970-a), Les villes. L'urbanisation (tome 1), Paris, Armand Colin.

Commissariat général du plan, (1970-b), Les villes. La société urbaine (tome 2 ), Paris, Armand Colin.

Debord G., (1967), La société du spectacle, Paris, Buchet-Chastel.

Devisme L. et al, (2000-a), La dimension territoriale de la centralité : de la commune à l'agglomération, Tours, Pôle d'initiative régional en Région Centre.

Devisme L., (2005), La ville décentrée. Figures centrales à l'épreuve des dynamiques urbaines, Paris, L'Harmattan.

Genestier P., (2001-a), « Des projets en paroles et en images. La rhétorique du projet face à la crise du vouloir politique », Espaces et Sociétés, n 105-106.

Genestier P., (2001-b), « Némésis et Nicodème ", Les Annales de la Recherche Urbaine, $n^{\circ} 90$

Guillaume M., (1977), « Les équipements collectifs source et mise en scène de pouvoir ", in Sfez L. (dir), L'objet local, Paris, UGE.
Joseph I., (2004), Météor. Les métamorphoses du métro, Paris, Economica.

Lefebvre H., (1960), « Les nouveaux ensembles urbains. Un cas concret : Lacq-Mourenx et les problèmes urbains de la nouvelle classe ouvrière ", Revue française de sociologie, I, (2).

Magnan R., Mathieu H., (1975), Orthopoles. Villes en îles, Paris, CRU.

Mangin D., (2004), La ville franchisée. Formes et structures de la ville contemporaine, Paris, La Villette.

Marie M., (1989), Les terres et les mots, Paris, Méridiens Klincksieck.

Marie M., (1995), « La guerre, la colonie, la ville et les sciences sociales », Sociologie du travail, n², pp. 277-299.

Ostrowetsky S., Bordreuil J.-S., (1975), Structure de communication et espace urbain La centralité, 2 tomes, EDRESS,

Université de Provence.

Ostrowetsky S., (1994), "Les centres urbains », in Ghorra-Gobin C. (dir), Penser la ville de demain. Qu'est-ce qui institue la ville ? Paris, L’Harmattan.

Ostrowetsky S., (2004), « Les villes nouvelles françaises : paris et apories. Esquisse d'une problématique ", Espaces et Sociétés, $n^{\circ} 4$.

Peron R., (1993), La fin des vitrines. Des temples de la consommation aux usines à vendre, Paris, ENS Cachan.

Vanoni D., Faure J., Auclair E., (2001), «Ville émergente : le fonctionnement de la multicentralité ", Recherche sociale, nº 157.

\section{Biographie}

LAURENT DEVISME est maître-assistant à l'École d'architecture de Nantes où il enseigne les sciences de l'espace des sociétés. Chercheur au LAUA (Ministère de la Culture), il s'intéresse notamment à l'action urbanistique contemporaine (en ses projets emblématiques comme en son action ordinaire) et à la visibilité métropolitaine. Il a coordonné le numéro 7 de la revue Lieux communs («Vertiges et prodiges de l'interdisciplinarité ») et prépare le numéro 8 avec Élisabeth Pasquier («Les échos du local »).

Il vient de publier La ville décentrée (L’Harmattan, 2005).

laurent.devisme@wanadoo.fr 